\title{
Inhibition of micro-ribonucleic acid-320 attenuates neurologic injuries after spinal cord ischemia
}

\author{
Fang He, MD, ${ }^{a}$ Enyi Shi, MD, PhD, ${ }^{b}$ Lihui Yan, MD,${ }^{c}$ Juchen Li, MD,${ }^{c}$ and Xiaojing Jiang, MD, $\mathrm{PhD}^{\mathrm{c}}$
}

\section{ABSTRACT}

Objective: Micro ribonucleic acids (miRNAs) are involved in a wide range of biological functions, in multiple tissues, including the central nervous system. We investigated a novel neuroprotective strategy of down-regulation of miR320 in the spinal cord under the condition of transient ischemia.

Methods: Spinal cord ischemia was induced in rats by cross-clamping the descending aorta for 14 minutes. Lentivirus vectors containing antisense oligonucleotides of rat miR-320 (antagomiR-320) were transfected into the experimental rats by means of intrathecal injection, 5 days before spinal cord ischemia. Control lentivirus vectors, or the vehicle, were given to the control animals. Hind-limb motor function was assessed during 48 hours after ischemia, using the Motor Deficit Index (MDI), and histologic examination was performed. In parallel experiments, expressions of miR-320, and the phosphorylation state of heat-shock protein 20 (phospho-Hsp20) in the spinal cord were evaluated by quantitative real-time polymerase chain reaction and western blot analysis.

Results: The time courses of expressions of miR-320 and phospho-Hsp20 in the spinal cord, after the transient ischemia, indicated that expression of phosphoHsp20 was negatively correlated with expression of miR-320. Transfection of antagomiR-320 significantly reduced expression of miR-320 in the spinal cord and dramatically up-regulated expression of phospho-Hsp20. Compared with controls, inhibition of miR-320 markedly improved hind-limb motor function, as evidenced by lower MDI scores, at $6,12,24$, and 48 hours after reperfusion, and increased the number of intact motor neurons in the lumbar spinal cord.

Conclusions: Inhibition of miR-320 induces neuroprotection in the spinal cord, against ischemia-reperfusion injury, possibly via up-regulation of phosphoHsp20. (J Thorac Cardiovasc Surg 2015;150:398-406)

From the ${ }^{\mathrm{a} D e p a r t m e n t}$ of Anesthesiology, Fourth Affiliated Hospital, and Departments of ${ }^{b}$ Cardiac Surgery and ${ }^{c}$ Anesthesiology, First Affiliated Hospital, China Medical University, Shenyang, People's Republic of China.

This work was supported by grant No. 81171175 from the National Natural Science Foundation of China, Beijing, People's Republic of China.

F. He and E. Shi contributed equally to the study.

Received for publication Jan 3, 2015; revisions received March 18, 2015; accepted for publication March 21, 2015; available ahead of print June 17, 2015.

Address for reprints: Xiaojing Jiang, MD, PhD, Department of Anesthesiology, First Affiliated Hospital, China Medical University, Shenyang, People's Republic of China 110001 (E-mail: jiangsophie@hotmail.com).

0022-5223/\$36.00

Copyright (c) 2015 by The American Association for Thoracic Surgery

http://dx.doi.org/10.1016/j.jtcvs.2015.03.066

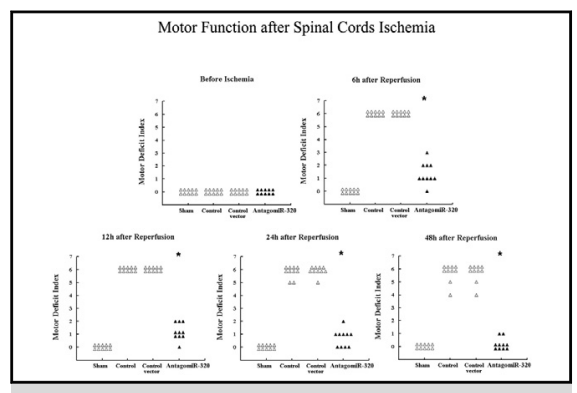

Inhibition of miR-320 protects motor function after spinal cord ischemia. AntagomiR-320, Antisense oligonucleotides of rat miR-320. ${ }^{\star} P<.01$, compared with the control group.

\section{Central Message}

Inhibition of miR-320, using transfection of lentivirus vectors containing antagomiR-320, induces neuroprotection, in rat spinal cord, against ischemia-reperfusion injury, possibly via up-regulation of phospho-Hsp20.

\section{Perspective}

A novel spinal cord protection strategy was performed; results indicate that down-regulation of miR-320 attenuates neurologic injury induced by transient spinal cord ischemia. Our findings expand understanding of miRNA function and demonstrate the critical role miRNAs play in spinal cord ischemia and reperfusion. A reasonable possibility is that miRNAs will become a novel therapeutic target for prevention of neurologic injury after thoracic aneurysm surgery.

See Editorial Commentary page 407.

Permanent or temporary spinal cord ischemia and consequent paraplegia or paralysis remain the most devastating complications after open and endovascular thoracoabdominal aortic aneurysm repair. Although the neurologic deficits have significantly decreased in recent times, with the progress of surgical adjuncts and pharmacologic interventions, this complication still cannot be prevented completely. ${ }^{1,2}$

Micro ribonucleic acids (miRNAs) are 21- to 23nucleotide non-protein-coding RNA molecules that act as negative regulators of gene expression, modulating the stability and/or the translational efficiency of target messenger RNAs. ${ }^{3}$ Ischemia induces profound changes in miRNA expression, and miRNAs play a pathogenetic role in 1 of 


\section{Abbreviations and Acronyms \\ antagomiR-320 $=$ antisense oligonucleotides of rat miR-320
MDI $=$ Motor Deficit Index
miRNA $=$ micro ribonucleic acid
phospho-Hsp20 = phosphorylation state of heat- shock protein 20
TU $\quad=$ transfection units

the various components of tissue response to ischemia. ${ }^{4}$ In addition, miRNAs play critical roles in brain ischemia and injury, neuroprotection, and neurodegeneration. ${ }^{5}$ Transient focal ischemia induces extensive temporal changes in rat cerebral microRNAome. ${ }^{6}$ Expression profiling even reveals that a large set of miRNAs is highly expressed in the adult mouse spinal cord.

Traumatic spinal cord injury induces dramatic changes of miRNAs expression. ${ }^{8}$ Existing studies demonstrate that spinal cord injury can induce extensive miRNA dysregulation that is parallel to the changes in mRNA expression and affects the key processes of spinal cord injury, including inflammation and apoptosis. Collectively, available findings suggest that the targeting of miRNA dysregulation is an attractive therapeutic strategy for spinal cord injury. ${ }^{9} \mathrm{How}-$ ever, little is known about the function of miRNAs in the process of ischemia-reperfusion injury of the spinal cord.

One member of the miRNA family, miR-320, plays functional roles in the differentiation of human mesenchymal stem cells, ${ }^{10}$ regulation of cardiac ischemia-reperfusion injury, ${ }^{11}$ and neuronal development. ${ }^{12}$ Furthermore, inhibition of miR-320a has been shown to induce neuroprotection against cerebral ischemia. ${ }^{13}$ However, the role of miR-320 in the ischemia-reperfusion injury of the spinal cord has not been investigated. The current study was therefore conducted to evaluate the effects of inhibition of miR-320 expression on neurologic outcomes in a rat model of spinal cord ischemia. Expression of heat-shock protein-20 (Hsp20), a possible target protein of miR-320 predicted by bioinformatics-based databases, was additionally explored.

\section{METHODS}

\section{Animals}

Male Wistar rats, weighing 230 to $270 \mathrm{~g}$, were used in the current study. The animal protocol was approved by the Ethics Review Committee for Animal Experimentation of China Medical University (Shenyang, People's Republic of China). It was conducted in accordance with the Guide for the Use and Care of Laboratory Animals (National Institutes of Health, Bethesda, Md).

\section{Lentivirus Vectors for Antisense Oligonucleotides of Rat miR-320}

Chemically modified antisense oligonucleotides of rat miR-320 (antagomiR-320) lentivirus gene transfer vector were constructed by Genechem (Shanghai, China). The recombinant lentivirus of antagomiR-320, and the control lentivirus without antagomiR-320, were prepared and titered to $1 \times 10^{9}$ transfection units (TU)/mL, according to manufacturer guidelines.

\section{Intrathecal Injection}

After anesthesia, animals were placed on an operating surface that flexed the animal's back. A human neonatal lumbar puncture needle of $25 \mathrm{G}$ was advanced into the spinal canal at the L4-L5 level. Proper placement of the needle in the intrathecal space was indicated by 3 signs: loss of resistance at the time of entry (tentative sign); tail flick (more definitive sign); and presence of cerebral spinal fluid in the needle hub (most definitive sign). Once correct needle placement was confirmed, the solutions were injected over the course of 1 minute. ${ }^{14}$ The animals were next placed head up for 30 minutes. The animals were included in the study only if they had normal hind-limb motor function 3 days after the intrathecal injection.

\section{Surgical Procedure}

Surgical preparation was conducted according to the method described previously. ${ }^{15}$ Rats were anesthetized with $10 \%$ chloral hydrate $(300 \mathrm{mg} / \mathrm{kg}$, intraperitoneally). Core body temperature was continuously monitored with a rectal probe, and was maintained at $37 \pm 0.5^{\circ} \mathrm{C}$ with the aid of a heating lamp. A tracheotomy was performed, and the animal was mechanically ventilated. A 24-gauge catheter was inserted into the tail artery to measure the distal blood pressure. Another 24-gauge catheter was inserted into the left carotid artery to measure the proximal blood pressure; this catheter was connected to an external blood reservoir, positioned at a height of $54 \mathrm{~cm}$ above the level of the rat's body, to maintain the mean proximal blood pressure at about $40 \mathrm{~mm} \mathrm{Hg}$ during aortic occlusion. ${ }^{16,17}$ Through a left thoracotomy, the descending aorta was exposed.

After systemic heparinization (200 IU/kg), spinal cord ischemia was induced by cross-clamping the descending aorta just distal to the left subclavian artery, for 14 minutes. The ischemia was confirmed by a reduction of the distal blood pressure to $<10 \mathrm{~mm} \mathrm{Hg}$. At the end of the procedure, the clamp was removed, and the blood in the external blood reservoir was reinfused. The chest was closed by layers. The cut of the trachea was closed carefully with a 6-0 suture after extubation.

\section{Experimental Protocol}

All rats except the sham animals were subjected to a 14-minute spinal cord ischemia. To measure expressions of miR-320 and phosphoHsp20 at various time points after reperfusion, 16 rats that had been subjected to the transient spinal cord ischemia were killed at 6,12 , 24 , and 48 hours after reperfusion, respectively $(n=4$, at each time point; Figure 1, A).

For neurologic injury study, rats were assigned to 4 groups randomly (Figure 1,B). Rats in the sham group received only the surgical procedure, without spinal cord ischemia. For the antagomiR-320 and control vector groups, approximately $1 \times 10^{7} \mathrm{TU}$ lentivirus vectors of antagomiR-320, or the control lentivirus vectors, respectively, in a total fluid volume of $10 \mu \mathrm{l}$, were intrathecally injected into each rat, 5 days before spinal cord ischemia. For the sham and control groups, the vehicle of the same volume, alone, was injected into each rat. In a parallel series of experiments, spinal cords were additionally collected 2 hours after reperfusion from the other 4 groups ( $\mathrm{n}=4$ per group; Figure $1, C$ ), to evaluate the effect of antagomiR320 on expressions of miR-320 and phospho-Hsp20.

\section{Neurologic Assessment}

Hind-limb motor function was assessed at 6, 12, 24, and 48 hours after reperfusion, using the Motor Deficit Index (MDI) score (quantified by ambulation and the placing/stepping reflex), ${ }^{16,17}$ by investigators who were blinded to group information. Hind-limb ambulation was graded as follows: 0, normal (symmetric and coordinated ambulation); 1 , toes flat under the body when walking, but ataxia present; 2, knuckle walking; 3 , 
Measurement of the Expression of miR-320 and phospho-Hsp20 at Different Time Points after Reperfusion

A

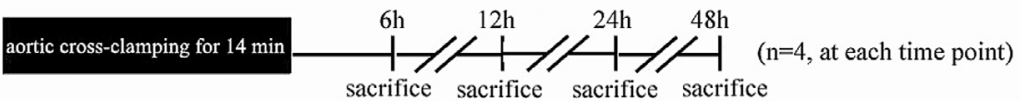

Neurological Injury Study

Sham

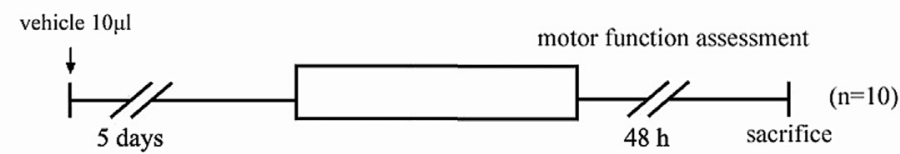

Control

vehicle $10 \mu \mathrm{l}$

Control

Controlvector

Control vector
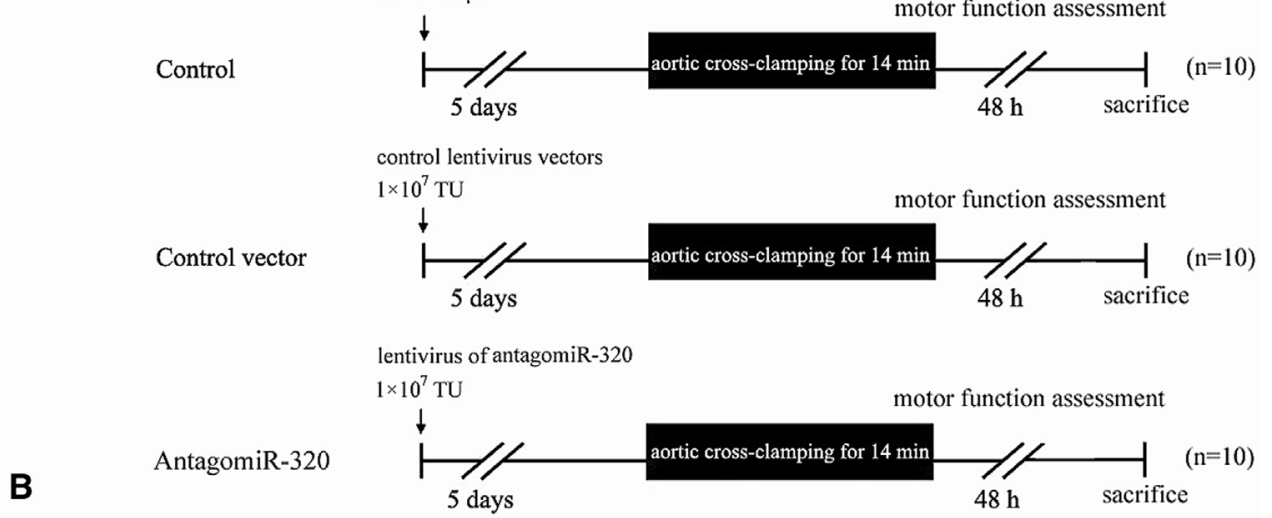

motor function assessment

Parallel Series for Measurement of the Expression of miR-320 and phospho-Hsp20 after Pretreatment with antagomiR-320

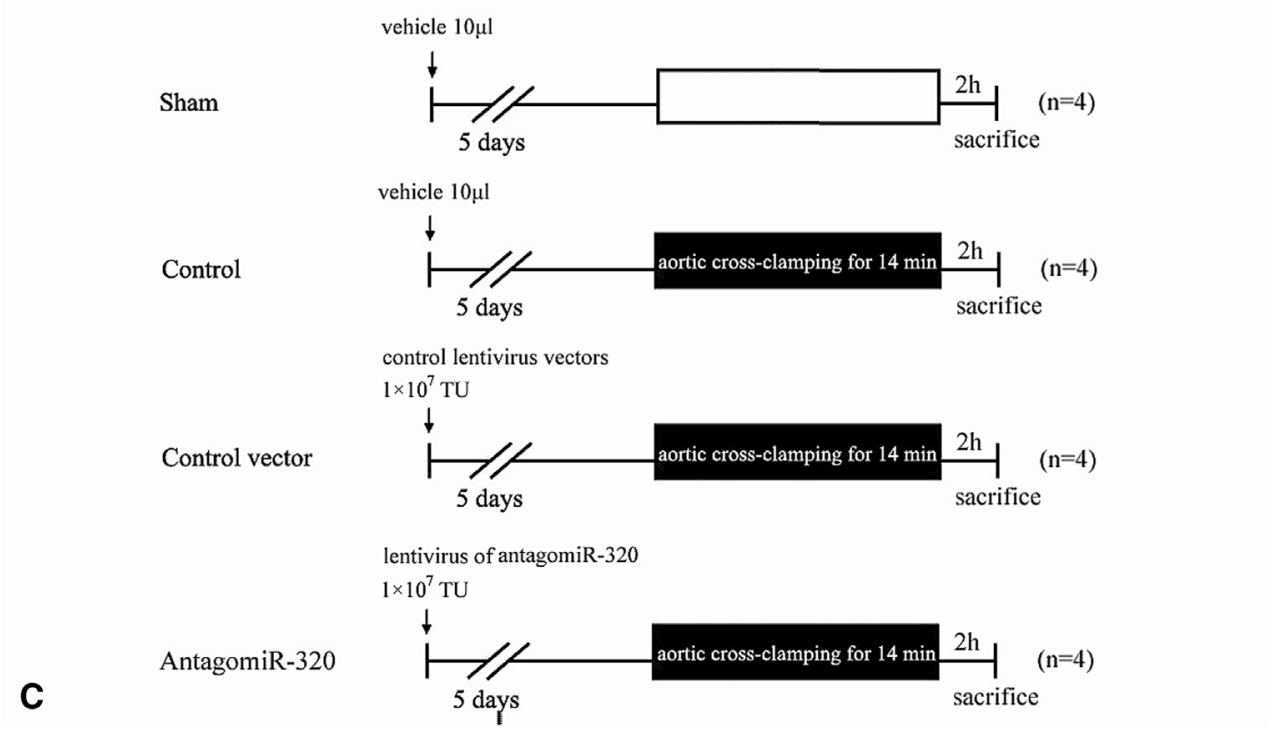

FIGURE 1. Experimental groups and protocol. miR-320, Micro-ribonucleic acid-320; phospho-Hsp20, phosphorylation state of heat-shock protein 20; $T U$, transfection units; antagomiR-320, antisense oligonucleotides of rat miR-320.

unable to knuckle walk but some movement of the hind limbs; and 4, no movement, or drags lower extremities. The placing/stepping reflex was assessed by the dragging movements and responses of the hind-paw dorsum when touching the floor surface. A coordinated lifting and placing response, which was generally evoked when a hind paw touched the ground, was graded as follows: 0, normal; 1, weak; and 2, no stepping. The MDI was calculated for each rat as the sum of these scores at each interval.

\section{Histologic Study}

For histologic study, rats were killed 48 hours after the transient spinal cord ischemia. Paraffin-embedded sections $(4 \mu \mathrm{m})$ of lumbar spinal cord (L4-L6) were stained with hematoxylin-eosin and Nissl dye. Gray-matter damage was assessed by counting the number of normal motor neurons in the ventral part of the gray matter (anterior to a transverse line drawn through the central canal), at the original magnification: $\times 400$. Cells that contained Nissl substance in the cytoplasm, loose chromatin, and 
prominent nucleoli were considered normal motor neurons. The number of normal motor neurons in each animal was obtained by averaging counts from 3 different slides stained with Nissl dye. ${ }^{16}$

\section{Extraction of miRNA, and Quantitative Real-Time Polymerase Chain Reaction}

Total RNA was isolated using Trizol reagent (Invitrogen, Carlsbad, Calif), according to manufacturer instructions. The first-strand complementary deoxyribonucleic acid was generated using the Reverse Transcription System Kit (Invitrogen). Real-time polymerase chain reaction was performed according to a standard protocol using a StepOne Plus system (Applied Biosystems, Foster City, Calif). The U6 served as an internal control. Changes in expression were determined by the $2^{-\Delta \Delta \mathrm{CT}}$ method. The primers used for miR-320 in the present study were as follows: forward primer: $5^{\prime}$ AAAAGCTGGGTTGAGAGGG-3'; RT primer: 5'-GTCGTATCCAGTGCGTGTCGTGG-AGTCGGCAATTGCACTGGATACGACTTCGCCCT-3'; reverse primer: 5'-TGCGTGTCGTGGAGTC-3'.

\section{Western Blot}

Phospho-Hsp20 was analyzed by sodium dodecyl sulfate polyacrylamide gel electrophoresis and immunoblotted with anti-Hsp20 (phospho S16) antibody (Abcom, Cambridge, Mass). The phospho-Hsp20 expression in each spinal cord tissue sample was analyzed with NIH Image (National Institutes of Health, Bethesda, Md) and quantified as a relative fold to the sham after normalization with $\beta$-actin.

\section{Statistical Analysis}

Parametric values are reported as mean $\pm \mathrm{SD}$. The Kruskal-Wallis test was used for nonparametric values (MDI and number of neurons), and the Mann-Whitney $U$ test was used as a posttest to identify specific differences between the groups. Parametric values were analyzed by 1-way or 2-way repeated measures (time and group) analysis of variance followed by unpaired Student $t$ tests with the Bonferroni correction.

\section{RESULTS}

\section{Expressions of phospho-Hsp20 and miRNA-320 After Reperfusion}

The time course of phospho-Hsp20 expression in spinal cords after reperfusion is illustrated in Figure 2, $A$ and $B$. The expression level of phospho-Hsp20 was markedly increased 6 hours after reperfusion, compared with the sham group (1.24 \pm 0.09 folds of the sham group, $P=$.036). After that, the expression of phospho-Hsp20 decreased slightly. Compared with the sham group, no significant differences were detected at the other 3 time points ( 1.14 $\pm 0.05,0.98 \pm 0.10$, and $0.99 \pm 0.08$ folds of the sham at 12 , 24 , and 48 hours after reperfusion, $P>.05$, respectively).

Expressions of miR-320 in the spinal cord at various time points after reperfusion are shown in Figure 2, $C$. The lowest expression of miR-320 appeared at 6 hours after reperfusion $(0.62 \pm 0.08$ folds of the sham group, $P=.042)$. After that point, the expression of miR-320 increased slightly. However, compared with the expression of miR320 of the sham group, no significant differences were found at the other 3 time points $(P>.05$, respectively).

\section{Expressions of phosphoHsp-20 and miR-320 After Transfection With antagomiR-320}

Compared with the control group, pretreatment with antagomiR-320 remarkably reduced the expression of
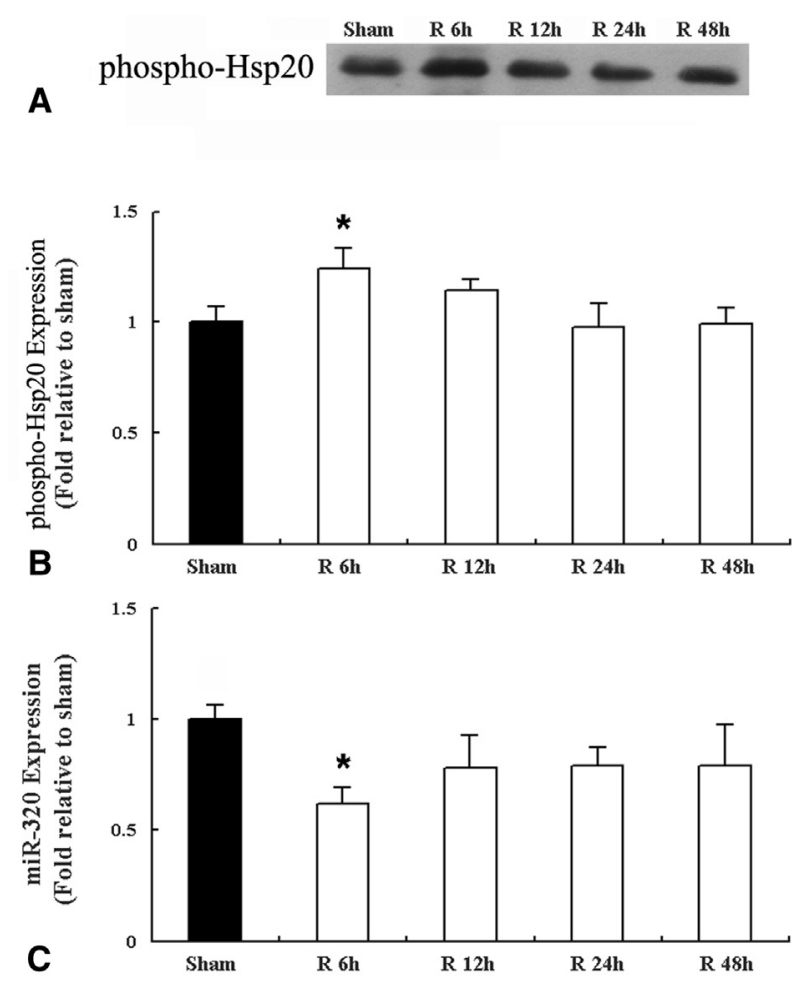

FIGURE 2. Time course of expressions of miR-320 and phospho-Hsp20 in the spinal cord after ischemia. A, Representative western blot picture showing phospho-Hsp20 expression. B, Densitometric quantification of phospho-Hsp20 expression. C, Densitometric quantification of miR-320 expression; $\mathrm{n}=4$ in each group. phospho-Hsp20, Phosphorylation state of heat-shock protein $20 ; m i R-320$, micro-ribonucleic acid-320. ${ }^{*} P<.01$, compared with the sham group.

miR-320 in the spinal cord; the results of quantitative real-time polymerase chain reaction evaluation are summarized in Figure $3, C(0.23 \pm 0.05$ vs $0.81 \pm 0.19$ folds of the sham group, $P=.002)$. In contrast, the control vectors had no effect on the expression level of miR-320 $(0.85 \pm 0.16 \mathrm{vs}$ $0.81 \pm 0.19$ folds of the sham group, $P=1.00$ ).

In spinal cords intrathecally injected with lentivirus of antagomiR-320, the phospho-Hsp20 level was dramatically increased, by approximately $80 \%$, compared with the control group, 2 hours after reperfusion $(1.89 \pm 0.20$ vs $1.1 \pm$ 0.14 folds of the sham group, $P=.001$; Figure $3, A$ and $B$ ). No marked enhancement of phospho-Hsp20 expression was detected in spinal cords that were intrathecally injected with control vectors ( $P=1.0$, compared with the control group).

\section{Mortality and Exclusion}

A total of 45 rats were enrolled in the protocol for neurologic injury study. Three rats were excluded because of death during the operation, or within 48 hours after the operation. Another 2 rats were omitted as a result of technical difficulties with the intrathecal injection. Complete data were obtained in the remaining 40 rats $(n=10$, for each of the 4 groups). 


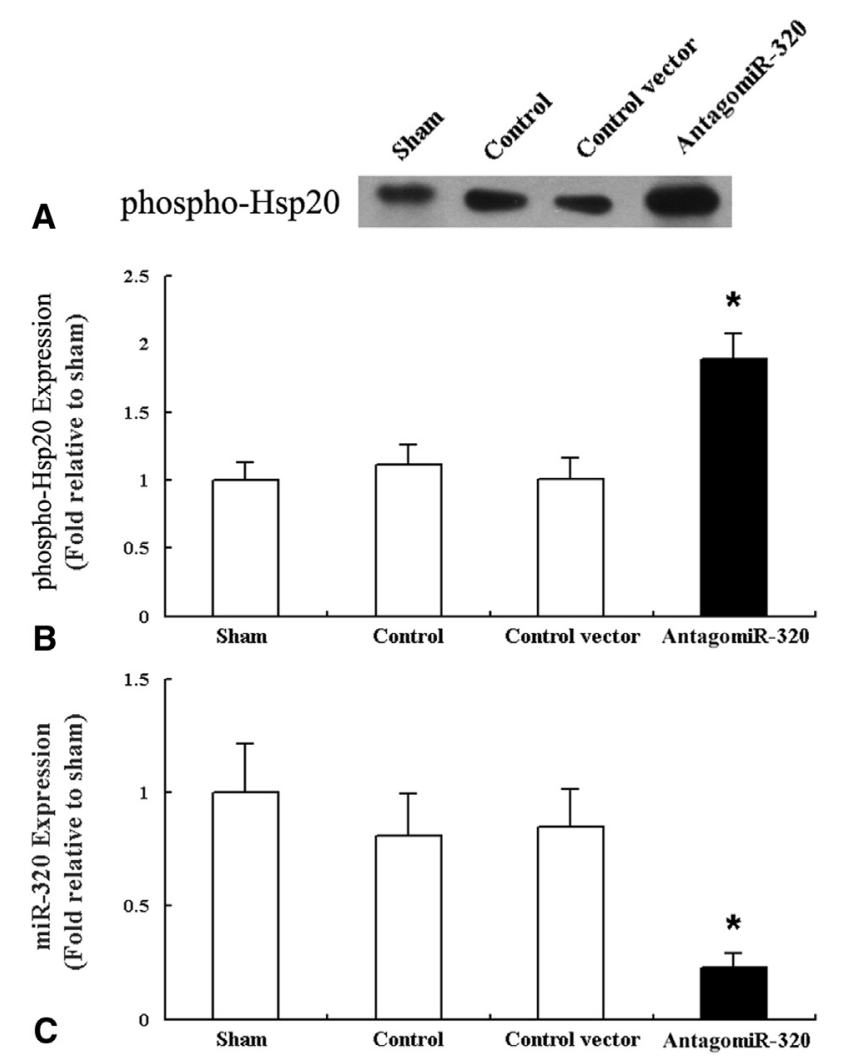

FIGURE 3. Effects of antagomiR-320 administration on expression of miR-320 and phospho-Hsp20 in the spinal cord, 2 hours after reperfusion. A, Representative western blot picture showing phospho-Hsp20 expression. B, Densitometric quantification of phospho-Hsp20 expression. C, Densitometric quantification of miR-320 expression; $n=4$ in each group. phospho-Hsp20, Phosphorylation state of heat shock protein 20; Antago$m i R-320$, antisense oligonucleotides of rat miR-320; miR-320, microribonucleic acid-320. $* P<.01$, compared with the control group.

\section{Physiologic Parameters}

Table 1 indicates the physiologic parameters from the 4 groups. No significant differences in the body weight or hemodynamic values of baseline were observed among the 4 groups. The rectal temperature was maintained at approximately $37^{\circ} \mathrm{C}$ in all animals, throughout the operation, and no significant differences were detected at any time point. During the aortic occlusion, the proximal mean blood pressure of all groups except the sham group was maintained at approximately $40 \mathrm{~mm} \mathrm{Hg}$, and the distal blood pressure was decreased to $<10 \mathrm{~mm} \mathrm{Hg}$. Compared with the baseline, the proximal and distal blood pressure and heart rate of the 3 groups receiving aortic occlusion were decreased after reperfusion $(P<.05$, respectively). No significant differences were found in the proximal and distal blood pressure and heart rate among the 3 groups receiving aortic occlusion, at any time point $(P>.05$, respectively).

\section{Neurologic Assessment}

The individual neurologic scores of the 4 groups, before the operation, and $6,12,24$, and 48 hours after reperfusion, are shown in Figure 4. All rats showed normal hind-limb motor function before the operation. Sham animals retained normal hind-limb motor function throughout the observation period. A 14-minute aortic occlusion resulted in severe hind-extremity neurologic deficits in both the control rats, and the rats receiving control vectors. By contrast, intrathecal injection of lentivirus of antagomiR-320 remarkably enhanced the motor function of hind limbs after spinal cord ischemia, as indicated by the significantly lower MDI scores of the antagomiR-320 group at the 4 observation time points after reperfusion (all $P<.01$ vs the control group, at the 4 time points after reperfusion). Equivalent

TABLE 1. Physiologic parameters

\begin{tabular}{|c|c|c|c|c|c|c|}
\hline Parameter & Sham & Control & Control vector & AntagomiR-320 & $\mathbf{F}$ & Significance \\
\hline Body weight (g) & $249 \pm 6$ & $253 \pm 10$ & $256 \pm 7$ & $251 \pm 6$ & 0.586 & 0.628 \\
\hline \multicolumn{7}{|l|}{ Rectal temperature $\left({ }^{\circ} \mathrm{C}\right)$} \\
\hline Baseline & $36.9 \pm 0.5$ & $37.1 \pm 0.6$ & $37.0 \pm 0.7$ & $37.1 \pm 0.4$ & (group) 0.132 & 0.941 \\
\hline Ischemia, $7 \mathrm{~min}$ & $37.0 \pm 0.5$ & $36.9 \pm 0.7$ & $36.9 \pm 0.6$ & $37.0 \pm 0.3$ & (time) 0.421 & 0.658 \\
\hline Reperfusion, $20 \mathrm{~min}$ & $37.1 \pm 0.3$ & $37.0 \pm 0.3$ & $37.1 \pm 0.4$ & $37.1 \pm 0.5$ & & \\
\hline \multicolumn{7}{|l|}{ Heart rate (beats/min) } \\
\hline Baseline & $339 \pm 19$ & $345 \pm 17$ & $343 \pm 23$ & $346 \pm 22$ & (group) 11.796 & 0.000 \\
\hline Ischemia, $7 \mathrm{~min}$ & $341 \pm 21$ & $263 \pm 25 *, \dagger$ & $256 \pm 27^{*}, \dagger$ & $272 \pm 28^{*}, \dagger$ & (time) 53.139 & 0.000 \\
\hline Reperfusion, 20 min & $344 \pm 15$ & $313 \pm 22 *, \dagger$ & $319 \pm 24^{*}, \dagger$ & $315 \pm 23^{*}, \dagger$ & & \\
\hline \multicolumn{7}{|c|}{ Proximal mean blood pressure (mm Hg) } \\
\hline Baseline & $89 \pm 6$ & $89 \pm 5$ & $90 \pm 7$ & $88 \pm 4$ & (group) 42.060 & 0.000 \\
\hline Ischemia, $7 \mathrm{~min}$ & $90 \pm 5$ & $41 \pm 2^{*}, \dagger$ & $40 \pm 2^{*}, \dagger$ & $40 \pm 1^{*}, \dagger$ & (time) 130.118 & 0.000 \\
\hline Reperfusion, $20 \mathrm{~min}$ & $90 \pm 3$ & $67 \pm 6^{*}, \dagger$ & $68 \pm 6^{*}, \dagger$ & $69 \pm 5^{*}, \dagger$ & & \\
\hline \multicolumn{7}{|c|}{ Distal mean blood pressure $(\mathrm{mm} \mathrm{Hg})$} \\
\hline Baseline & $85 \pm 5$ & $86 \pm 6$ & $85 \pm 7$ & $84 \pm 5$ & (group) 36.446 & 0.000 \\
\hline Ischemia, $7 \mathrm{~min}$ & $86 \pm 4$ & $4 \pm 1^{*}, \dagger$ & $3 \pm 1^{*}, \dagger$ & $4 \pm 1^{*}, \dagger$ & (time) 158.720 & 0.000 \\
\hline Reperfusion, $20 \mathrm{~min}$ & $87 \pm 3$ & $64 \pm 6^{*}, \dagger$ & $63 \pm 5^{*}, \dagger$ & $65 \pm 5^{*}, \dagger$ & & \\
\hline
\end{tabular}

$\mathrm{N}=10$ for each group. AntagomiR-320, Antisense oligonucleotides of rat miR-320. $* P<.05$, versus the sham group. $\dagger P<.05$, versus baseline of the same group. 

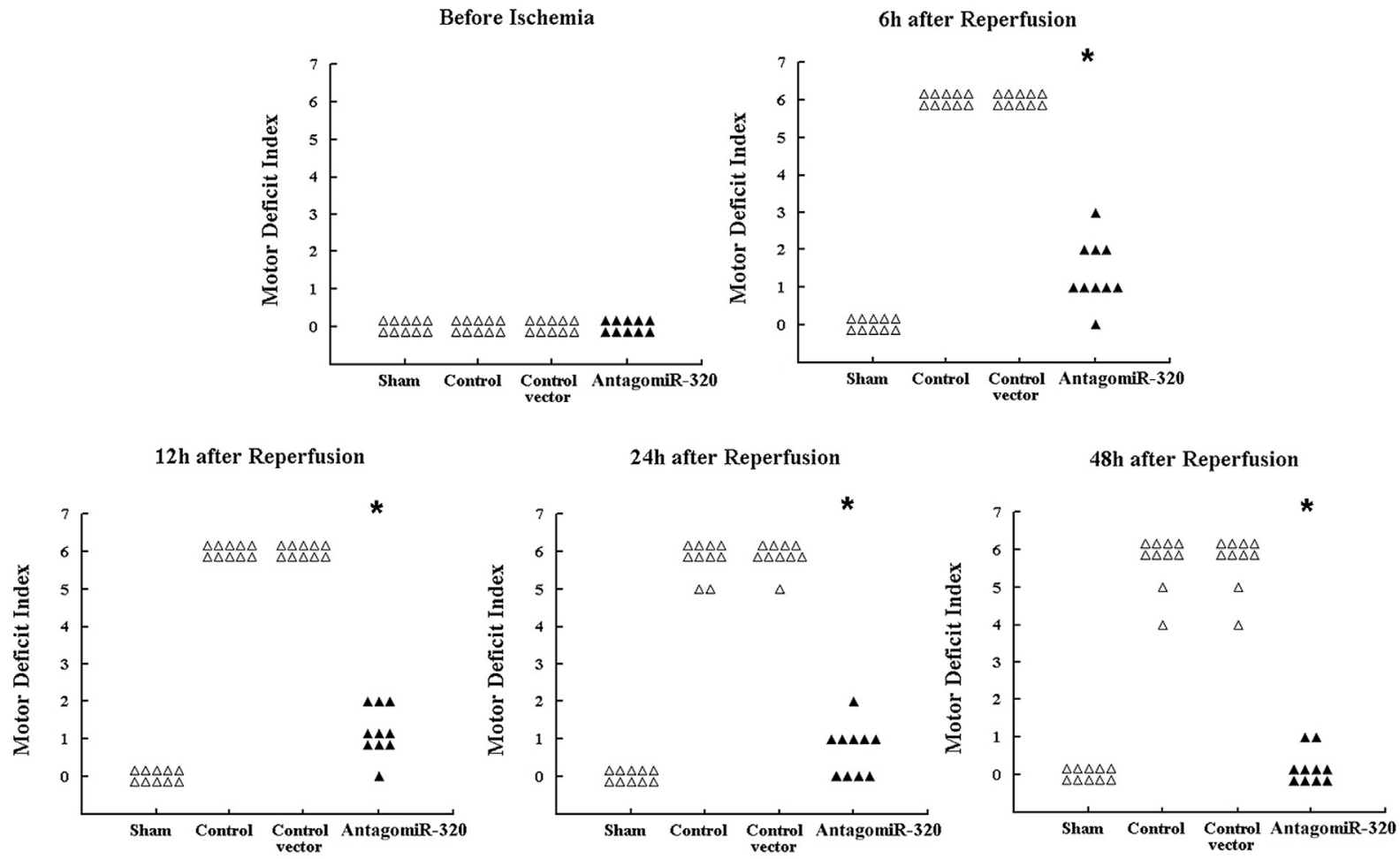

FIGURE 4. Effects of antagomiR-320 administration on hind-limb motor function, assessed using the Motor Deficit Index, before spinal cord ischemia, and $6,12,24$, and 48 hours after reperfusion. Solid triangles indicate the group of antagomiR-320. ${ }^{*} P<.01$, compared with the control group. AntagomiR320, Antisense oligonucleotides of rat miR-320.

MDI scores were observed in the control group and the control vector group, at the 4 observation time points after reperfusion (all $P>.05$ ).

\section{Histologic Assessment}

Representative sections of lumbar spinal cord stained with hematoxylin-eosin and Nissl dye are shown in Figure 5, $A$, and the results of counting normal motor neurons are summarized in Figure 5, B. In animals who underwent sham operations, the spinal cord was intact, and many large motor neurons were present in the anterior horn. Severe neurologic damage was readily detected in animals of the control group 48 hours after spinal cord ischemia, as evidenced by vacuolization, frank necrosis, and an almost total loss of motor neurons. No significant difference was found in the intact motor neuron count between the control group and the control vector group $(P=1.00)$. In contrast, slighter histologic changes were found in the lumbar spinal cord of animals in the antagomiR-320 group, and the intact motor neurons were preserved to a much greater extent than those of the control group $(P<.01)$.

\section{DISCUSSION}

In a well-established rat model of transient spinal cord ischemia, ${ }^{15}$ the current study indicated that inhibition of miR-320, a spinal cord enriched miRNA, regulated the expression of phospho-Hsp20 and enhanced the neurologic outcomes after spinal cord ischemia. This report is the first to indicate that protection of the spinal cord against ischemia-reperfusion injury can be conducted by regulation of miRNAs.

Neuron death and apoptosis are the key cellular events in the ischemic central nervous system. A well-established finding is that multiple genes and their target proteins are responsible for such pathologic processes after ischemia and reperfusion. Because miRNAs are endogenous negative regulators of gene expression, it is reasonable that miRNAs are involved in ischemia-reperfusion injury of the central nervous system.

A few studies have assessed the role of miRNAs in various models of cerebral ischemia. By targeting a proapoptotic BCL2 family member, astrocyte-enriched miR29a-induced neuroprotection on the hippocampal CA1 delayed neuronal death after forebrain ischemia. ${ }^{18}$ In another rat model of forebrain ischemia, inhibition of miRNA-181a, by pretreatment with antagomiR, reduced astrocyte dysfunction and increased CA1 neuronal survival. ${ }^{19}$ Down-regulation of miR-181b was additionally indicated to induce neuroprotection against ischemic injury, via negative regulation of HSPA5 and UCHL1 protein levels. ${ }^{20}$ The brain-abundant miRNA-124 was shown to promote neuronal survival under ischemic conditions, 

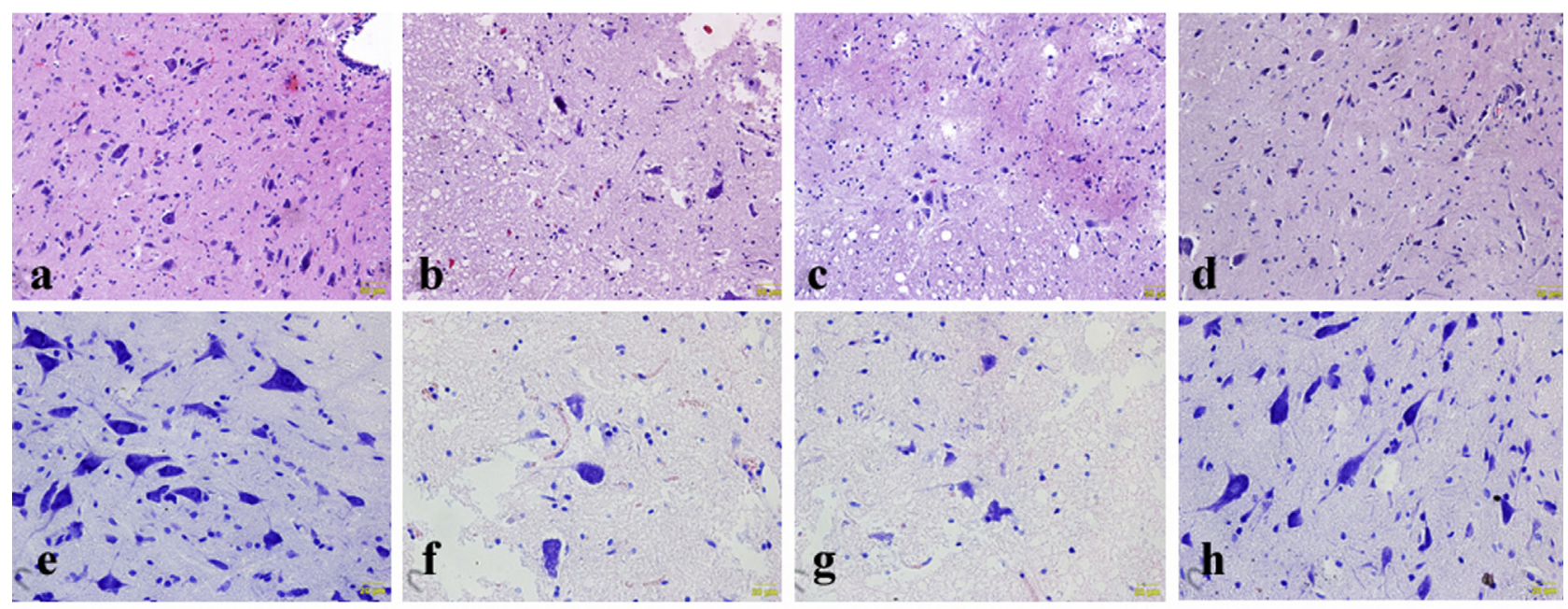

A

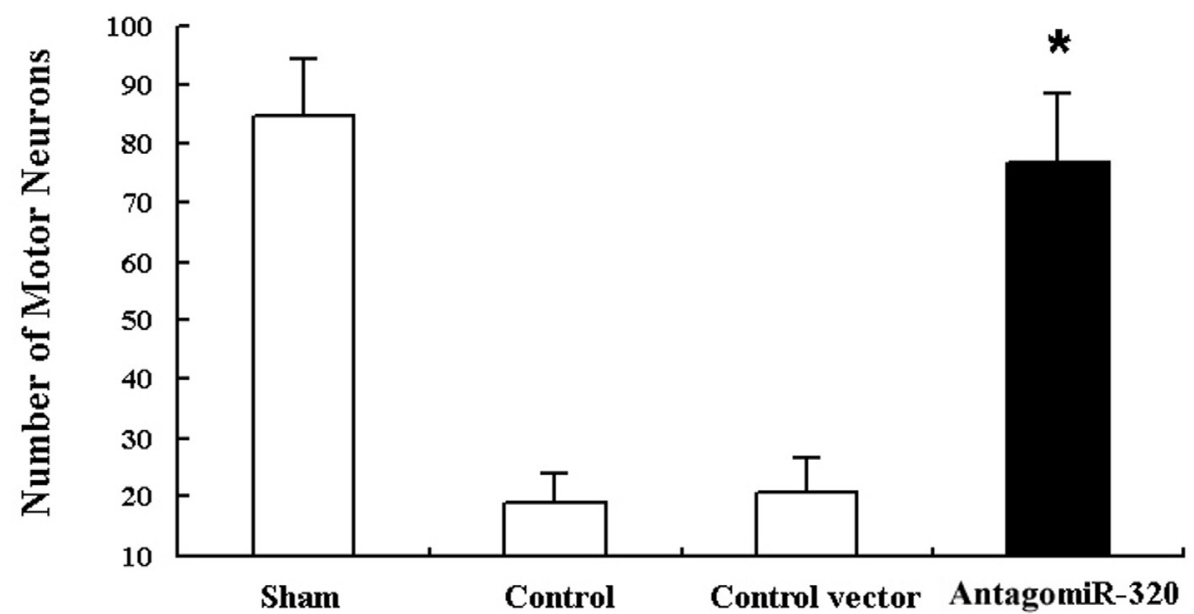

FIGURE 5. Effects of antagomiR-320 administration on neuron survival after spinal cord ischemia. A, Representative sections of lumbar spinal cord, stained with hematoxylin-eosin (a-d) and Nissl dye (e-h): sham group (a, e); control group (b, f); control vector group (c, g); antagomiR-320 group (d, h). B, Number of large motor neurons in the ventral gray matter of lumber spinal cord. AntagomiR-320, Antisense oligonucleotides of rat miR-320. $* P<.01$, compared with the control group.

via Usp14-dependent REST degradation. ${ }^{21}$ Anti-miR-320a was shown to bring about a reduction of infarct volume in cerebral ischemia, with a concomitant increase in aquaporins 1 and 4 mRNA and protein expressions. ${ }^{13}$

However, little is known about the function of miRNAs in ischemia-reperfusion injury of spinal cord. In the current study, miR-320 was confirmed to be enriched in the spinal cord. Inhibition of miR-320 expression by pretreatment of antagomiR-320 significantly attenuated the neuron loss, in lumbar spinal cord, induced by the transient ischemia, and improved the motor function of hind limbs. In addition, the data suggest that miR-320 is a negative regulator of neuroprotective effects against ischemia-reperfusion injury of spinal cord.

To elucidate the potential mechanism of miR-320 in the regulation of ischemia-reperfusion injury of spinal cord, possibilities for the target protein were explored. With the help of bioinformatics-based databases, ${ }^{22,23}$ and using computational predictions, as detailed at TargetScan (http://genes.mit.edu/targetscan/), the search was narrowed down to Hsp20 as a possible target protein of miR-320. In murine hearts, miR-320 was involved in the regulation of cardiac injury, induced by ischemia-reperfusion and dysfunction, via antithetical regulation of Hsp20. Decreased endogenous miR-320 expression reduced cardiomyocyte apoptosis when it was induced by simulated ischemiareperfusion; overexpression of miR-320 increased sensitivity to cell death triggered by ischemia-reperfusion. ${ }^{11}$

In the current study, the time courses were measured, of expressions of miR-320 and phospho-Hsp20 in spinal cord, after ischemia and reperfusion. The results showed that the expression levels of phospho-Hsp20 were negatively correlated with the expressions of miR-320 in the spinal cord. Furthermore, inhibition of miR-320 by transfection of 
antagomiR-320 in vivo dramatically elevated the expression of phospho-Hsp20 in the spinal cord. Taken together, these data indicate that miR-320 expression is correlated with phospho-Hsp20 levels in the spinal cord, and miR-320 acts as a negative regulator of phospho-Hsp20 expression.

As a member of the small heat-shock protein family, Hsp20 (also called HspB6) performs many physiologic functions, including maintenance of the cellular cytoskeleton, regulation of protein aggregation, and modulation of cell survival in several tissues and cells. As a cardioprotective protein, the role of Hsp20 has been well established in the heart, under ischemic conditions. Its functions include antiapoptosis effects, ${ }^{24}$ reduction of the infarct area, ${ }^{11,24,25}$ and enhancement of contractile function. ${ }^{24}$ In addition, Hsp20 is highly and continually expressed in the brain and has been implicated in the disease process of the central nervous system. ${ }^{26}$ In cultured rat hippocampal neurons, expression of Hsp20 was up-regulated under sodium arsenite, oxidative, or hyperosmotic stress, and acted to protect neurons from the stress conditions. ${ }^{27}$ Overexpression of Hsp20 protected cultured mouse neuroblastoma cells from oxygen-glucose deprivation and reoxygenation injury, by inhibition of apoptosis, via mitochondrial pathways. ${ }^{28}$

In the current study, continuous expression of phosphoHsp20 was detected in spinal cord after ischemia. Furthermore, overexpression of phospho-Hsp20 was accompanied by decreased motor neuron loss, and improvement of neurologic function of spinal cord suffering from transient ischemia. These data indicate that up-regulation of phosphoHsp20 may induce a powerful neuroprotection against ischemia-reperfusion injury of the spinal cord.

Several limitations of this study merit comment. First, the mode of action for miR-320 regulation of phospho-Hsp20 is not well established in the current study. Further investigation is needed to identify whether other target proteins of miR-320, aside from Hsp-20, contribute to the neuroprotections observed in the current study. Second, although severe neurologic injury was induced in the current rat model, the aortic cross-clamping time was much shorter than that used in clinical practice. Because of the anatomic difference in blood supply to the spinal cord, the rat model of spinal cord ischemia may not reliably mimic spinal cord ischemia in clinical practice. In addition, strategies used in clinical practice, such as hypothermia and lumbar cerebrospinalfluid drainage, were not used in our model.

In conclusion, precautionary measures to protect the spinal cord are necessary and feasible. A novel protective strategy for the spinal cord was performed, and the results indicate that down-regulation of miR-320 attenuates neurologic injuries induced by transient spinal cord ischemia, via an Hsp20 pathway. Our findings expand the understanding of the function of miRNAs and demonstrate for the first time that miRNAs play a critical role in spinal cord ischemia. By extension, miR-320 may become a novel therapeutic target for prevention of neurologic injury after thoracic aneurysm surgery.

\section{Conflict of Interest Statement}

Authors have nothing to disclose with regard to commercial support.

\section{References}

1. Gkremoutis A, Schmandra T, Meyn M, Schmitz-Rixen T, Keese M. Hybrid approach to emergent and urgent treatment of complex thoracoabdominal aortic pathology. Eur J Vasc Endovasc Surg. 2014;48:407-13.

2. Preventza O, Cervera R, Cooley DA, Bakaeen FG, Mohamed AS, Cheong BY, et al. Acute type I aortic dissection: traditional versus hybrid repair with antegrade stent delivery to the descending thoracic aorta. J Thorac Cardiovasc Surg. 2014;148:119-25.

3. Kim VN, Han J, Siomi MC. Biogenesis of small RNAs in animals. Nat Rev Mol Cell Biol. 2009;10:126-39.

4. Fasanaro P, Greco S, Ivan M, Capogrossi MC, Martelli F. MicroRNA: emerging therapeutic targets in acute ischemic disease. Pharmacol Ther. 2012;125: 92-104.

5. Saugstad JA. MicroRNAs as effectors of brain function with roles in ischemia and injury, neuroprotection, and neurodegeneration. J Cereb Blood Flow Metab 2010;30:1564-76.

6. Dharap A, Bowen K, Place R, Li LC, Vemuganti R. Transient focal ischemia induces extensive temporal changes in rat cerebral microRNAome. J Cereb Blood Flow Metab. 2009;29:675-87.

7. Bak M, Silahtaroglu A, Møller M, Christensen M, Rath MF, Skryabin B, et al. MicroRNA expression in the adult mouse central nervous system. RNA. 2008; 14:432-44.

8. Liu NK, Wang XF, Lu QB, Xu XM. Altered microRNA expression following traumatic spinal cord injury. Exp Neurol. 2009;219:424-9.

9. Ning B, Gao L, Liu RH, Liu Y, Zhang NS, Chen ZY. microRNAs in spinal cord injury: potential roles and therapeutic implications. Int J Biol Sci. 2014;10: 997-1006.

10. Hamam D, Ali D, Vishnubalaji R, Hamam R, Al-Nbaheen M, Chen L, et al. microRNA-320/RUNX2 axis regulates adipocytic differentiation of human mesenchymal (skeletal) stem cells. Cell Death Dis. 2014;5:e1499.

11. Ren XP, Wu J, Wang X, Sartor MA, Qian J, Jones K, et al, MicroRNA-320 is involved in the regulation of cardiac ischemia/reperfusion injury by targeting heat-shock protein 20. Circulation. 2009;119:2357-66.

12. White RE, Giffard RG. MicroRNA-320 induces neurite outgrowth by targeting ARPP-19. Neuroreport. 2012;23:590-5.

13. Sepramaniam S, Armugam A, Lim KY, Karolina DS, Swaminathan P, Tan JR, et al. MicroRNA 320a functions as a novel endogenous modulator of aquaporins 1 and 4 as well as a potential therapeutic target in cerebral ischemia. J Biol Chem. 2010;285:29223-30.

14. Judas GI, Ferreira SG, Simas R, Sannomiya P, Benício A, da Silva LF, et al Intrathecal injection of human umbilical cord blood stem cells attenuates spinal cord ischemic compromise in rats. Interact Cardiovasc Thorac Surg. 2014;18: 757-62.

15. LeMay DR, Neal S, Neal S, Zelenock GB, D’Alecy LG. Paraplegia in the rat induced by aortic cross-clamping: model characterization and glucose exacerbation of neurologic deficit. J Vasc Surg. 1987;6:383-90.

16. Saito T, Saito S, Yamamoto H, Tsuchida M. Neuroprotection following mild hypothermia after spinal cord ischemia in rats. J Vasc Surg. 2013; 57:173-81.

17. Taira Y, Marsala M. Effect of proximal arterial perfusion pressure on function, spinal cord blood flow, and histopathologic changes after increasing intervals of aortic occlusion in the rat. Stroke. 1996;27:1850-8.

18. Ouyang YB, Xu L, Lu Y, Sun X, Yue S, Xiong XX, et al. Astrocyte-enriched miR-29a targets PUMA and reduces neuronal vulnerability to forebrain ischemia. Glia. 2013;61:1784-94.

19. Moon JM, Xu L, Giffard RG. Inhibition of microRNA-181 reduces forebrain ischemia-induced neuronal loss. J Cereb Blood Flow Metab. 2013;33: 1976-82.

20. Peng Z, Li J, Li Y, Yang X, Feng S, Han S, et al. Downregulation of miR-181b in mouse brain following ischemic stroke induces neuroprotection against ischemic injury through targeting heat shock protein A5 and ubiquitin carboxyl-terminal hydrolase isozyme L1. J Neurosci Res. 2013;91:1349-62. 
21. Doeppner TR, Doehring M, Bretschneider E, Zechariah A, Kaltwasser B, Müller B, et al. MicroRNA-124 protects against focal cerebral ischemia via mechanisms involving Usp14-dependent REST degradation. Acta Neuropathol. 2013;126:251-65.

22. Lewis BP, Shih IH, Jones-Rhoades MW, Bartel DP, Burge CB. Prediction of mammalian microRNA targets. Cell. 2003;115:787-98.

23. Friedman RC, Farh KK, Burge CB, Bartel DP. Most mammalian mRNAs are conserved targets of microRNAs. Genome Res. 2009;19:92-105.

24. Fan GC, Ren X, Qian J, Yuan Q, Nicolaou P, Wang Y, et al. Novel cardioprotective role of a small heat-shock protein, Hsp20, against ischemia/reperfusion injury. Circulation. 2005;111:1792-9.

25. Fan GC, Zhou X, Wang X, Song G, Qian J, Nicolaou P, et al. Heat shock protein 20 interacting with phosphorylated Akt reduces doxorubicin-triggered oxidative stress and cardiotoxicity. Circ Res. 2008;103:1270-9.
26. Quraishe S, Asuni A, Boelens WC, O'Connor V, Wyttenbach A. Expression of the small heat shock protein family in the mouse CNS: differential anatomical and biochemical compartmentalization. Neuroscience. 2008; 153:483-91.

27. Bartelt-Kirbach B, Golenhofen N. Reaction of small heat-shock proteins to different kinds of cellular stress in cultured rat hippocampal neurons. Cell Stress Chaperones. 2014;19:155-8.

28. Zeng L, Tan J, Hu Z, Lu W, Yang B. Hsp20 protects neuroblastoma cells from ischemia/reperfusion injury by inhibition of apoptosis via a mechanism that involves the mitochondrial pathways. Curr Neurovasc Res. 2010;7: 281-7.

Key Words: spinal cord, ischemia, miRNA

Readers who found these articles interesting may also like to read the following papers found in recent and future issues of our sister publications, Seminars in Thoracic and Cardiovascular Surgery and Operative Techniques in Thoracic and Cardiovascular Surgery!

Evolving Technology/Basic Science

State of the Art: RichardWeisel. The Promise and Challenges of Cardiac Stem Cell Therapy. Semin Thorac Cardiovasc Surg. Spring 2014;26(1):44-52. 